\title{
EFEITOS DO FATOR DE CRESCIMENTO DE FIBROBLASTO BÁSICO (FCFß) NA CICATRIZAÇÃO DE ANASTOMOSES DO ESÔFAGO
}

\section{EFFECTS OF FIBROBLAST GROWTH FACTOR-BETA (FCF $\beta$ ) ON ESOPHAGEAL ANASTOMOSIS HEALING}

\author{
Aldo da Cunha Medeiros, TCBC-RN ${ }^{1}$ \\ Henrique José da Mota, TCBC-RN ${ }^{2}$ \\ Tertuliano Aires Neto, TCBC-RN ${ }^{2}$ \\ Antônio Medeiros Dantas Filho, TCBC-RN ${ }^{2}$ \\ Lidiane Maria de Brito Macedo ${ }^{3}$ \\ Nara Medeiros Cunha de Melo ${ }^{3}$
}

\begin{abstract}
RESUMO: Objetivo: Os fatores de crescimento são substâncias moduladoras do processo de cicatrização. O fator de crescimento de fibroblastos básico (FCF $\beta$ ) liberado pelas plaquetas, macrófagos e pelos próprios fibroblastos, estimulam a proliferação celular, a produção de colágeno e de outros elementos da matriz celular, favorecendo o processo da cicatrização, mesmo em situações adversas, como diabetes e uso de corticosteróides. O presente estudo objetivou determinar a influência do FCF $\beta$ no processo de cicatrização de anastomoses esofageanas em modelo de experimentação animal, avaliando-se a resistência à pressão, formação de tecido de granulação e deposição de colágeno. Método: Foram estudados dois grupos A e B, ambos com 10 ratos de linhagem Wistar, separados de forma aleatória, todos submetidos à secção e anastomose do esôfago por via abdominal. Nos animais do grupo A, foi feita aplicação tópica na linha de sutura de 10ng de FCF $\beta$. No grupo B (controle) foi aplicado igual volume de solução salina. Os animais foram sacrificados no $7^{\circ}$ dia, o esôfago ressecado para teste de resistência da anastomose, estudo qualitativo do aporte de células inflamatórias, da angiogênese e quantificação do colágeno na zona da anastomose, através de sistema digital. Resultados: A densidade média dos parâmetros histológicos do grupo A foi $9095,51 \pm 1284,5$, maior que no grupo B, que teve densidade 7162,4 $\pm 1273,19(\mathrm{p}=0,013)$. A resistência da anastomose do grupo A teve a média $210 \pm 18,88 \mathrm{mmHg}$, significativamente maior que no grupo $B$, que atingiu o valor $157 \pm 29,55 \mathrm{mmHg}(\mathrm{p}=0,0024)$. Conclusão: Este estudo concluiu que o FCF $\beta$ atuou melhorando a cicatrização e aumentando significativamente a resistência de anastomoses do esôfago realizadas em ratos.
\end{abstract}

Descritores: Cicatrização de feridas; Fator 2 de crescimento de fibroblasto; Colágeno; Esôfago; Anastomose cirúrgica.

\section{INTRODUÇÃO}

A cicatrização de feridas é um processo contínuo e ordenado de coagulação, inflamação e reparo, que envolve uma série de fatores de crescimento. Eles regulam a infiltração inflamatória, proliferação celular, a ativação e interferência na cicatrização das feridas, na dependência do fator envolvido ${ }^{1}$. A resposta imediata a qualquer dano tecidual é a formação de um coágulo de fibrina e ativação plaquetária². As

1. Professor Adjunto, Doutor em Cirurgia, Chefe do Núcleo de Cirurgia Experimental e da Disciplina de Técnica Operatória-UFRN; Pesquisador nível I do CNPq.

2. Professor do Departamento de Cirurgia-UFRN; Aluno do Programa de Pós-Graduação nível doutorado.

3. Alunos bolsistas de Iniciação Científica PIBIC-CNPq.

Recebido em 04/09/2002

Aceito para publicação em 10/12/2002

Trabalho realizado no Núcleo de Cirurgia Experimental-UFRN; Apoiado pelo CNPq. 
plaquetas, ao se desgranularem, também liberam citocinas e Fator de Crescimento de Fibroblastos (FCF) que, atuando nos tecidos lesados, promove migração e proliferação, resultando em uma resposta inflamatória local. Neutrófilos, linfócitos e macrófagos são as células especificamente envolvidas nesta inflamação. Os macrófagos produzem FCF, que estimula a proliferação de fibroblastos e a formação de colágeno. Os fibroblastos também liberam FCF para efetivar a renovação do colágeno depositado no ferimento, promover a contração da ferida e aumentam a síntese de proteoglicanos, componentes da matriz extra-celular, por até seis meses após o dano tecidual $^{3}$. A formação da matriz e a fase de remodelação também dependem do FCF, ativado pelas células endoteliais e dos epitélios, contribuindo igualmente com a angiogênese, reepitalização e proliferação celular. Os fibroblastos e as células endoteliais liberam FCF que ajudam na coordenação dos estágios finais da cicatrização $0^{3,4}$.

Duas formas de fator de crescimento de fibroblastos são conhecidas: a alfa ou ácida (FCF$\alpha)$ e a beta ou básica (FCF- $\beta$ ). São diferentes apenas quanto ao pH ótimo de atuação destas substâncias, sendo o beta mais ativo nos fenômenos da cicatrização. Estudos in vitro comprovaram que os FCF têm propriedades angiogênicas ${ }^{5,6}$, mitogênicas $^{7}$, estimulam a diferenciação celular ${ }^{4}$, induzem a formação dos componentes da matriz extra-celular ${ }^{7}$ e promovem a estruturação de tecidos em reparo ${ }^{5}$. Na pele, foi demonstrada uma melhora nas propriedades biomecânicas de retalhos após aplicação de FCF $\beta^{8}$. Feridas em animais diabéticos, em uso de corticosteróides e na presença de infecção, têm apresentado aumento da angiogênese, proliferação de fibroblastos e deposição de colágeno significativamente mais satisfatórios que nos controles. Estudos clínicos em que o FCF foi usado em úlceras crônicas de decúbito mostraram resultados satisfatórios ${ }^{9}$.

O esôfago caracteriza-se por ser um órgão de difícil manipulação cirúrgica e que está muito freqüentemente associado a complicações como fístulas e deiscências de anastomoses, que complicam com infecções graves. Essa propensão às complicações se dá pela irrigação sangüínea segmentar e deficiente, disposição predominantemente longitudinal das fibras musculares de sua parede, a ausência de serosa, problemas da técnica operatória, entre ou$\operatorname{tros}^{10}$. Dados da literatura revelam incidência de fístulas após esofagectomia desde $15,5 \%{ }^{11}$ até $54 \%{ }^{12}$, caracterizando as anastomoses esofageanas como de alto risco.

Tomando por base os dados acima, foi realizado estudo experimental com o objetivo de testar a ação do FCF- $\beta$ em aplicação tópica em anastomoses do esôfago, observando-se os possíveis benefícios do fator na evolução da cicatrização do órgão. Foi avaliada sua ação quanto à resistência à pressão, formação de tecido de granulação, angiogênese e deposição de colágeno na zona de anastomose.

\section{MÉTODO}

Foi utilizado o Fator de Crescimento de Fibroblasto de reação básica (FCF- $\beta$ ) estabilizado em heparina, humano, recombinante, de procedência SIGMAâ. Foram usados 20 ratos da linhagem Wistar pesando $275 \pm 17 \mathrm{~g}$, separados aleatoriamente em dois grupos: $A(n=10)$ e $B(n=10)$. Observados em gaiolas individuais, com controle de temperatura, partículas e ciclo de 12 horas claro-escuro, durante todo o experimento receberam água e alimento Labina-Purina ${ }^{\circledR}$ ad libitum. No dia anterior à operação os animais permaneceram 12 horas recebendo apenas água. Após anestesia com pentobarbital sódico, na dose de $20 \mathrm{mg} / \mathrm{Kg}$, por via intraperitoneal, foi feita epilação da região abdominal, contenção na mesa de operações em decúbito dorsal e antissepsia com PVPI 1\%. Uma laparotomia mediana de $4 \mathrm{~cm} \mathrm{a}$ partir do apêndice xifóide deu acesso ao esôfago, que foi clampeado com pinça de Satinsky, exteriorizado, seccionado transversalmente e anastomosado com fio de polipropineno 6-0 em pontos simples separados, com auxílio de microscópio cirúrgico. Nos animais do grupo A foi feita a aplicação tópica na linha de sutura de 10ng de FCF- $\beta$ em veículo constituído de uma preparação viscosa e aderente segundo a técnica utilizada por Slavin $e t$ $a l^{13}$. Nos animais do grupo B (controle) foi aplicado o mesmo volume de solução salina sobre as suturas. O abdome foi fechado com nylon 4-0 em dois planos de sutura e no $7^{\circ}$ dia de observação os animais receberam dose letal de anestésico. O esôfago foi ressecado para realização de teste de resistência da anastomose à pressão, de acordo com técnica previamente descrita ${ }^{14}$. Feito o teste, a zona de anastomose foi avaliada através de exame histológico. 
As peças foram fixadas em formol $10 \%$ por 48 horas, processadas e coradas com hematoxilinaeosina e tricrômio de Masson. A análise foi feita através de microscopia óptica, num microscópio Olympusâ modelo BX50, utilizando-se o aumento de 200x, sendo as imagens captadas através de câmera digital, e enviadas ao computador para estudo da densidade média dos componentes histológicos da cicatrização, através do programa Image Pro Plus ${ }^{\circledR}$, versão 4.0 para Windows ${ }^{\circledR}$. O aporte de fibras colágenas, de células inflamatórias inerentes ao processo cicatricial, e a formação de neovasos, foram quantificados em unidades de densidade média. Foram analisados dez campos microscópicos de cada lâmina, num total de duas lâminas por animal.

Os dados obtidos foram analisados através do teste t de Student, utilizando-se o programa Bioestat 2.0 windows, considerando-se significativo quando a diferença entre as médias correspondeu a um $\mathrm{p}<0,05$.

\section{RESULTADOS}

A análise histológica demonstrou um maior aporte de macrófagos, neutrófilos, linfócitos e fibroblastos, com maior formação do tecido de granulação e de fibras de colágeno no Grupo A, onde foi utilizado o FCF- $\beta$ (Figura 1). No grupo B, este afluxo de células inflamatórias e colágeno mostrouse de menor intensidade que nos animais estudados no Grupo A (Figura 2). No que diz respeito à angiogênese, foi observada uma maior presença de vasos neo-formados no Grupo A, quando comparado com o grupo B (Figura 3).

Após a análise digital, que mediu a densidade média da área ocupada pelo colágeno, células inflamatórias e neovasos em relação à área total dos campos microscópicos, constatou-se a predominância de maiores densidades nas amostras do Grupo A, com uma média de $9095,51 \pm 1284,5$, contra uma média de $7162,40 \pm 1273,19$ observada no Grupo B ( $\mathrm{p}=0,013)$. Os dados constatados estão relacionados na Tabela 1.

A análise da resistência das anastomoses mostrou que elas suportaram sem complicações o período de observação. Nos animais do grupo A (experimental) a zona de anastomose do esôfago suportou pressão de insuflação que variou de 180 a 240 $\mathrm{mmHg}$, que correspondeu à média $210 \pm 18,88 \mathrm{mmHg}$. No grupo B (controle) a pressão suportada variou de 120 a $210 \mathrm{mmHg}$, com média $157 \pm 29,55 \mathrm{mmHg}$. Quando foram comparadas as médias das resis- tências à pressão dos dois grupos, observou-se uma diferença significante, com $\mathrm{p}=0,0024$ (Tabela 2).

Tabela 1 - Resultados obtidos através da análise digital de cortes histológicos das zonas de anastomoses de ambos os grupos. Representam a densidade média do colágeno e células inflamatórias em dez campos microscópios de cada duas lâminas por animal.

\begin{tabular}{lcc}
\hline Animal n $^{\mathbf{0}}$ & Grupo A (FCF $\boldsymbol{\beta})$ Grupo B (Controle) \\
\hline 1 & 9835,21 & 6598,22 \\
2 & 6730,50 & 8412,25 \\
3 & 8025,90 & 7026,88 \\
4 & 9035,45 & 5065,40 \\
5 & 10248,56 & 6146,27 \\
6 & 11076,20 & 9021,50 \\
7 & 9587,15 & 6976,30 \\
8 & 7830,45 & 8890,22 \\
9 & 8856,10 & 7212,90 \\
10 & 9733,48 & 6278,24 \\
\hline Média & 9095,51 & 7162,40 \\
Desvio padrão & 1284,50 & 1273,19 \\
\hline
\end{tabular}

T calculado $=3,04$.

$(p=0,013)$ Diferença significante.

Tabela 2 - Resultados dos testes de resistência da linha de sutura. Representam as pressões, em $\mathrm{mmHg}$, suportadas pelas anastomoses dos animais do grupo experimental (em uso de FCF- $\beta$ ) e do grupo de controle.

\begin{tabular}{lcc}
\hline Animal n $^{\mathbf{0}}$ & Grupo A (FCF- $\boldsymbol{\beta})$ & Grupo B (Controle) \\
\hline 1 & 230 & 120 \\
2 & 190 & 180 \\
3 & 195 & 175 \\
4 & 180 & 145 \\
5 & 240 & 155 \\
6 & 215 & 210 \\
7 & 225 & 130 \\
8 & 200 & 185 \\
9 & 210 & 145 \\
10 & 215 & 125 \\
\hline Médias \pm desvio padrão & $210 \pm 18,88$ & $157 \pm 29,55$ \\
\hline
\end{tabular}

T calculado $=4,16$.

$(p=0,0024)$ Diferença significante. 


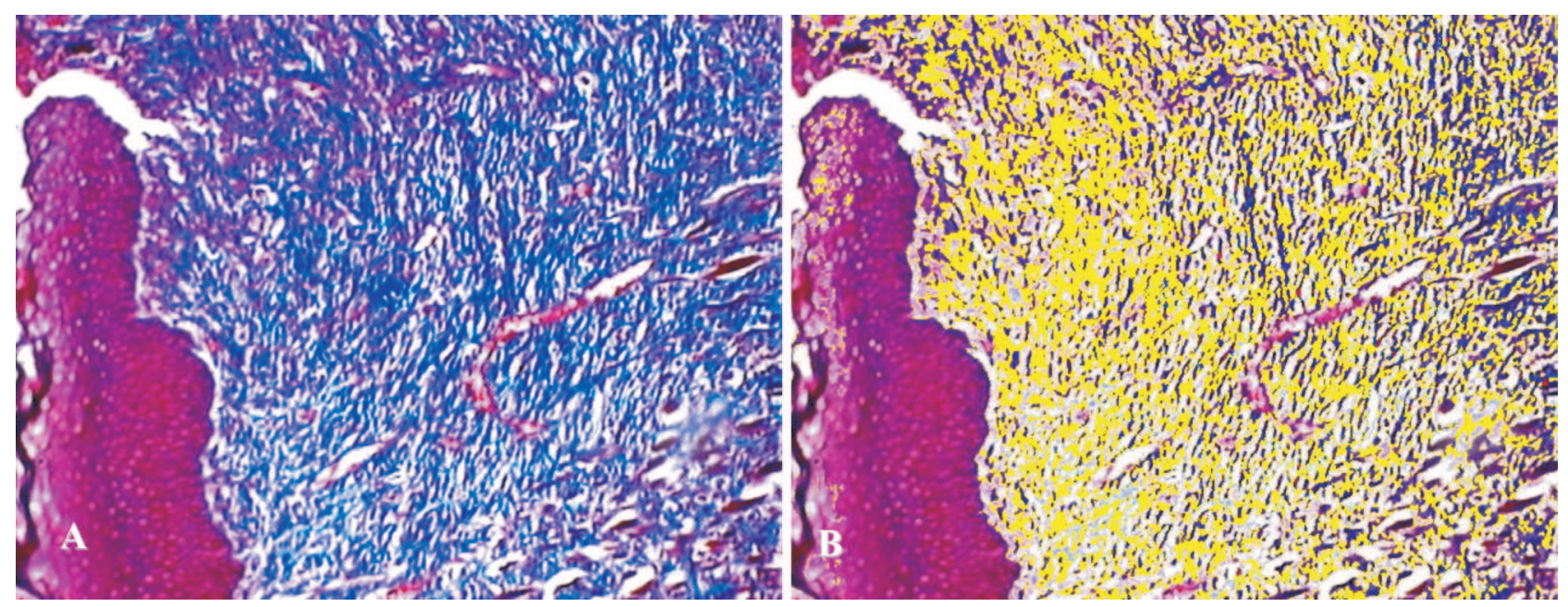

Figura 1 - Análise histológica computadorizada de anastomose do Grupo A. A: Submucosa esofágica com grande quantidade de fibras colágenas (em azul). É possível observar um tecido de granulação abundante e numerosos vasos neo-formados. B: Colágeno selecionado em amarelo através do sistema digital, para efeito de quantificação da sua densidade média. 200x.
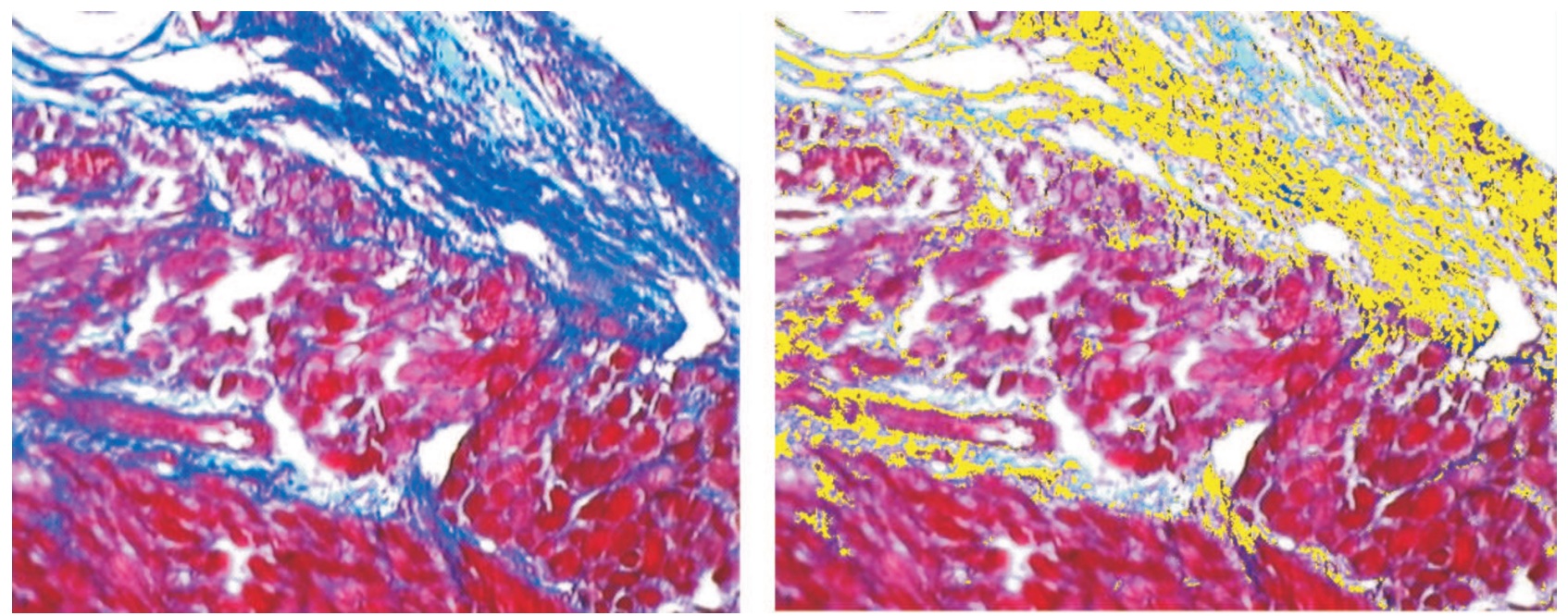

Figura 2 - Análise histológica computadorizada de anastomose do Grupo B. A: Acúmulo de fibras colágenas na lesão cirúrgica esofágica (em azul). B Colágeno selecionado em amarelo através do sistema digital, para efeito de quantificação da densidade média. Constata-se menor quantidade de colágeno que na figura 1, além de menores quantidades de células inflamatórias e vasos neoformados.
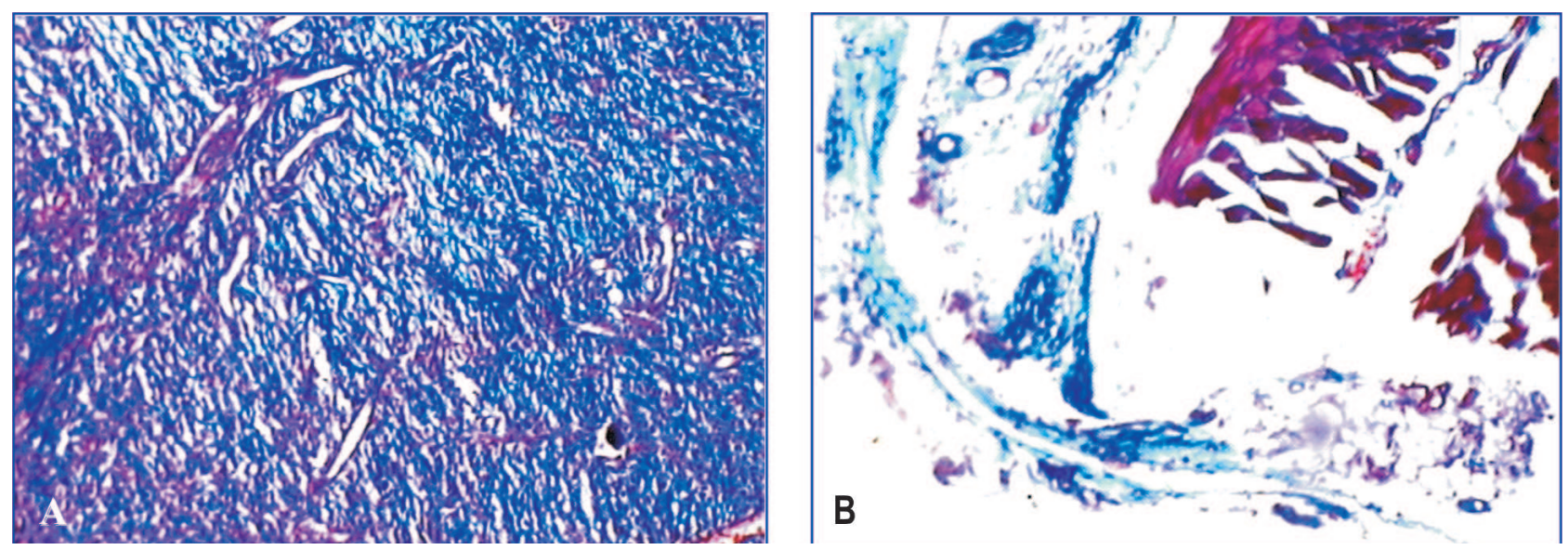

Figura 3 - Em A: Amostra da ferida operatória do esôfago de animal tratado com FCF- $\beta$, (grupo A) mostrando expressiva angiogênese. $B$ : Área cicatricial da parede esofagiana, com poucos vasos formados a partir do processo inflamatório (grupo B). 


\section{DISCUSSÃO}

Nas operações praticadas no esôfago o grande temor dos cirurgiões decorre da alta incidência de fístulas e deiscência de sutura, especialmente quando as anastomoses são praticadas no esôfago do segmento torácico ${ }^{11}$. As fístulas esofágicas são tipicamente complicações que ameaçam a vida, por causa dos órgãos localizados adjacentes ao esôfago, acometendo mais comumente o sistema respiratório. São raras as fístulas esofago-cutâneas, com exceção daquelas fístulas em casos de anastomoses na região do esôfago cervical ${ }^{12,15}$. A cirurgia é uma das causas principais, mas pode ocorrer em intubação prolongada, traumatismo contuso ou penetrante, endoscopia e dilatação esofágica ${ }^{11}$. O reconhecimento e o tratamento precoces desse tipo de fístula são de primordial importância, pois podem causar mediastinite, pneumonia, abscesso pulmonar e derrame pleural. Os pacientes portadores de fístulas esofágicas não reconhecidas podem evoluir rapidamente para sepse, falência de múltiplos órgãos e sistemas e morte ${ }^{15}$.

No presente trabalho não foram evidenciadas fístulas esofageanas, entretanto, as anastomoses que receberam tratamento tópico com FCF $\beta$ suportaram pressão, até a ruptura, maior do que o grupo de controle, fazendo com que se possa inferir a sua influência na maior resistência à pressão.

OFCF $\beta$ tem apresentado resultados satisfatórios em estudos de cicatrização in vitro $e$ in vivo. Ficou demonstrada a melhora na cicatrização do esterno, mesmo após a ligadura das artérias torácicas internas ${ }^{16}$. Em um dos raros estudos em que foi empregado o FCF $\beta$ em anastomoses digestivas, ficou demonstrado que este fator reverteu a ação depressora do corticosteróide na cicatrização do intestino de ratos $^{13}$. No presente trabalho pôde ser observado o aumento da deposição de colágeno, melhor evolução dos parâmetros histológicos e maior resistência à pressão nas anastomoses, quando se fez o uso tópico do FCF $\beta$. Outra constatação importante foi o significativo aumento na proliferação de neovasos na zona de anastomose do esôfago dos animais em uso de FCF $\beta$, fato que tem sido demonstrado em vários estudos ${ }^{5,6}$. Uma vez ocorrendo intensa neovascularização na zona de anastomose, e consequientemente maior aporte de sangue e oxigênio, todos os demais eventos do processo de cicatrização estarão otimizados. Em relato de revisão foi descrito o uso clínico e experimental do FCF $\beta$ em um número expressivo de situações, a maioria com resultados animadores ${ }^{17,18}$. Embora a farmacocinética dos FCF esteja para ser estabelecida, os resultados dos estudos em animais sugerem um potencial benefício terapêutico em várias situações clínicas e cirúrgicas de dificuldades para cirurgiões e pacientes ${ }^{18}$. No trabalho aqui descrito, tornou-se evidente o benefício do FCF $\beta$ nas anastomoses do esôfago de ratos.

Os dados disponíveis permitiram a conclusão de que o FCF $\beta$ promoveu um maior aporte de células inflamatórias, um aumento na angiogênese, incremento na deposição de colágeno, além do aumento na resistência à pressão em anastomoses do esôfago terminal de ratos.

\begin{abstract}
Background: Growth factors are substances that modulates tissue healing. Fibroblast growth factor is produced by platelets, macrofages and fibroblasts itself, stimulates cellular proliferation, collagen production and other elements of the cellular matrix, enhancing the healing proccess, even under adverse conditions. In order to study the influence of the basic Fibroblast Growth Factor (FCF-b) on the healing of esophageal anastomosis, an experimental model was produced to measure the anastomosis strenght, the amount of collagen and granulation tissue. Methods: Twenty adult Wistar rats weighing $275 \pm 17 \mathrm{~g}$, were randomly allocated into two groups of ten each: Group A and B, were submitted to esophageal transection and anastomosis through a laparotomy approach. The anastomosis suture line of the group A was treated with a topical solution containing 1Ong of FCF-b, whereas the control group $(B)$ was treated with saline. Animals were killed on the $7^{\text {th }}$ postoperative day, and the esophageal specimen removed to evaluate the anastomosis strenght, the input of inflamatory cells, and the amount of angiogenesis and collagen layered at the suture line. A digital system was used to process the histologic study. Results:Group A showed greater amounts of inflamatory cells, angiogenesis and collagen density, as compared with the control group B. The quantitative mean density of the hystological data reached $9095.51 \pm 1284.5$ in Group A and $7162.40 \pm 1273.19$ in group $B(p=0.013)$. The anastomosis suture line resistance to blowout was $210 \pm 18.88 \mathrm{mmHg}$ in group $A$ and $157 \pm 29.55 \mathrm{mmHg}$ in group $B(p=0.0024)$. Conclusion: This experimental model in rats confirms that FCF-b, when applied topically, enhances healing of esofageal anastomosis.
\end{abstract}

Key Words: Wound healing; Fibroblast Growth Factor 2; Collagen; Esophagus; Anastomosis, surgical. 


\section{REFERÊNCIAS}

1. Herndon DN, Hayward PG, Rutan RL, et al. - Growth hormones and factors in surgical patients. Adv Surg, 1992, 25:65-97.

2. Cromack DT, Porras-Reyes B, Mustoe TA - Current concepts in wound healing: growth factor and macrophage interaction. J Trauma, 1990, 30(12 suppl): S129-133.

3. McGrath $\mathrm{MH}$ - Peptide growth factors and wound healing. Clin Plast Surg, 1990, 17(3):421-432.

4. Schweigerer L - Basic fibroblast growth factor as a wound healing hormone. Trends Pharmacol Sci, 1988, 9(12):427-428.

5. Folkman J, Klägsbrun M - Angiogenic factors. Science, 1987, 235(4787):442-447.

6. Ferrara N - Vascular endothelial growth factor and the regulation of angiogenesis. Recent Prog Horm Res, 2000, 55:15-35.

7. Gosporadowicz D - Fibroblast growth factor. Chemical structure and biologic function. Clin Orthop, 1990, 257:231-248.

8. Lu WW, Ip WY, Jin WM, et al. - Biomechanical properties of thin skin flap after basic fibroblast growth factor (bFGF) administration. Br J Plast Surg, 2000, 53(3):225-229.

9. Davidson JM, Broadley KN - Manipulation of the wound-healing process with basic fibroblast growth factor. Ann N Y Acad Sci, 1991, 638:306-315.

10. Wright C, Gaissert HA, Puma F, et al. - "Benign and malignant tumors of esophagus". In Morris PJ, Malt RA (eds) - Oxford textbook of surgery. Oxford, 1995, pp. 893-904.
11. Shahian DM, Neptune WB, Ellis FH, et al. Transthoracic versus extrathoracic esophagectomy: mortality, morbidity and long term survival. Ann Thorac Surg, 1986, 41(3):237-246.

12. Hankins JR, Attar S, Coughlin TR Jr, et al. - Carcinoma of the esophagus: a comparison of the results of transhiatal versus transthoracic resection. Ann Thorac Surg, 1989, 47(5):700-705.

13. Slavin J, Nash JR, Kingsnorth AN - Effect of transforming growth factor beta and basic fibroblast growth factor on steroid-impaired healing intestinal wounds. Br J Surg, 1992, 79(1):69-72.

14. Medeiros AC, Ramos CCF, Freire TMGL, et al. - Uso de novo adesivo cirúrgico em anastomoses do colo: estudo experimental em ratos. Acta Cir Bras, 1990, 5(4):136-140.

15. Ginsberg RJ, Cooper JD - Esophageal fistula. World J Surg, 1983, 7(4):455-462.

16. Iwakura A, Tabata Y, Miyao M, et al. - Novel method to enhance sternal healing after harvesting bilateral internal thoracic arteries with the use of basic fibroblast growth factor. Circulation, 2000, 102(19 suppl 3):S307311.

17. Nugent MA, Iozzo RV - Fibroblast growth factor-2. Int J Biochem Cell Biol, 2000, 32(2):115-120.

18. Graham A-The use of growth factors in clinical practice. J Wound Care, 1998, 7(10):536-540.

Endereço para correspondência: Aldo da Cunha Medeiros

Av. Miguel Alcides Araújo 1889 59078-270 Natal-RN 